\title{
Top-down design for a system to control operant choice experiments
}

\author{
SANDRA S. HALE and JOEL MYERSON \\ University of California, Riverside, California 92521 \\ and \\ FRANCIS M. MIEZIN \\ California Institute of Technology, Pasadena, California 91125
}

\begin{abstract}
STATIONS, a FORTRAN program to control operant choice experiments, was developed based on the principles of top-down design and structured programming. As a consequence, the program consists of modules organized in a hierarchical fashion. Separate modules deal with different types of reinforcement schedules, such as variable ratio (VR) and variable interval (VI). These modules produce specific schedules with different parameters by using sequences of values read in at run time and then randomly sampled. This form of organization permits modifications to be made via changes confined to one module without global repercussions. The clarity of structure that results from the application of top-down design and structured programming should enable future users to more easily adapt this package to suit their specific needs.
\end{abstract}

The variety of mini- and microcomputers available today for the laboratory setting gives rise to a variety of installations and implementations. In the face of this diversity, software portability becomes a key issue. The term "portability," as we use it, refers to (1) whether or not a program can be implemented on different machines and (2) whether or not different programmers can adapt a program to suit their needs. Attaining portability is a general problem. To see how this general problem can be solved, this paper describes how a specific solution, a flexible, portable software package, was developed. The principles used in the development of this software are well established and deal with top-down design and structured programming (Graham, 1979).

One way to obtain portability between machines is to write software in a standard high-level language. FORTRAN 66 is the standard version of FORTRAN on which all later versions are based. More important, a program written in FORTRAN 66 may be compiled on a system capable of handling a later version, although the reverse is not true. BASIC, on the other hand, does not have a standard form; virtually all implementations of BASIC have different capabilities and/or limitations. Although PASCAL has a standard form, it tends to

This work was supported by National Science Foundation Grant BNS 80-11567 to J. Myerson. Requests for reprints should be sent to Sandra S. Hale, Department of Psychology, University of California, Riverside, California 92521. Requests for copies of documented software should be sent to Joel Myerson, Department of Psychology, Cardinal Stritch College, Milwaukee, Wisconsin 53217. differ slightly from installation to installation and is not always available or supported for a given computer.

Portability between programmers depends on those characteristics of software that result in ease of comprehension of the program structure, ease of modification, and ease of debugging new implementations or modifications. It is common to try to achieve some clarity in programs by thorough commenting; however, this is usually insufficient (Brooks, 1974).

Top-down design and structured programming also address the problems of program clarity and ease of modification. Top-down design requires the programmer to think in terms of modules organized in a hierarchical fashion. A specific program is developed by proceeding from the highest level (the mainline) down through the lower levels of nested subroutines and eventually down to the deepest level of routines (primitives). The modular organization provides a coherence that enables the programmer to pinpoint the level and module to be modified. Furthermore, any modification made in one module tends to have few, if any, repercussions in other modules either at the same level or at higher levels.

Whereas top-down design yields clarity at a macrolevel, structured programming tends to yield clarity at the microlevel (i.e., organization within modules). Structured programming, in the field of computer science, refers to the use of control statements to achieve coherent and efficient program modules (Graham, 1979; Kernigan \& Plauger, 1976). For programmers who have recently acquired formal training in the field of computer science, these two approaches 
are the foundation on which all other skills are built. However, for many psychologists who have acquired programming techniques informally as the need to solve specific problems arises, these techniques are not necessarily part of their software skills.

Portable programs can prevent duplication of effort by different programmers. They can also prevent wasted effort on the part of individual programmers. Modular organization can greatly simplify the debugging process. Moreover, old programs can be as incomprehensible to the original programmer as to any other person. Structural clarity can therefore greatly aid the person who must later modify his/her program. Our experience has also taught us the benefits of modular organization for team programming, whereby two programmers can concurrently work on different portions of the same package. Coherent well structured modules become software tools to be reused in later packages (Kernigan \& Plauger, 1976).

The problem we faced was how to control operant choice experiments. This problem has been solved before (cf. SKED); however, within the constraints of our system, we set about finding a solution to this problem that would also provide the benefits described above Although our STATIONS package is intended to be highly portable, it is important to describe the environment for which it was originally intended. The system is based on a Data General Nova computer with a DOS operating system, one that does not provide foreground/background capabilities. A digital I/O board (MDB Systems) provides 16 channels for input and 16 channels for output. The board has been modified to include several additional features that are required by the method chosen for handling digital input data. Every time the occurrence of a real-world event is signaled to the computer, a 16-bit word is passed in which each bit signals whether or not an event has just occurred on a separate input channel. This method of handling input necessitated the following modifications: (1) Additional gates (detail logic) were added to indicate the occurrence of events; (2) logic was added to tell the assembly language package when there is information to be collected and set an interrupt; and (3) logic was added for strobing the detail logic (i.e., to sample all 16 input channels and pass the information to the CPU).

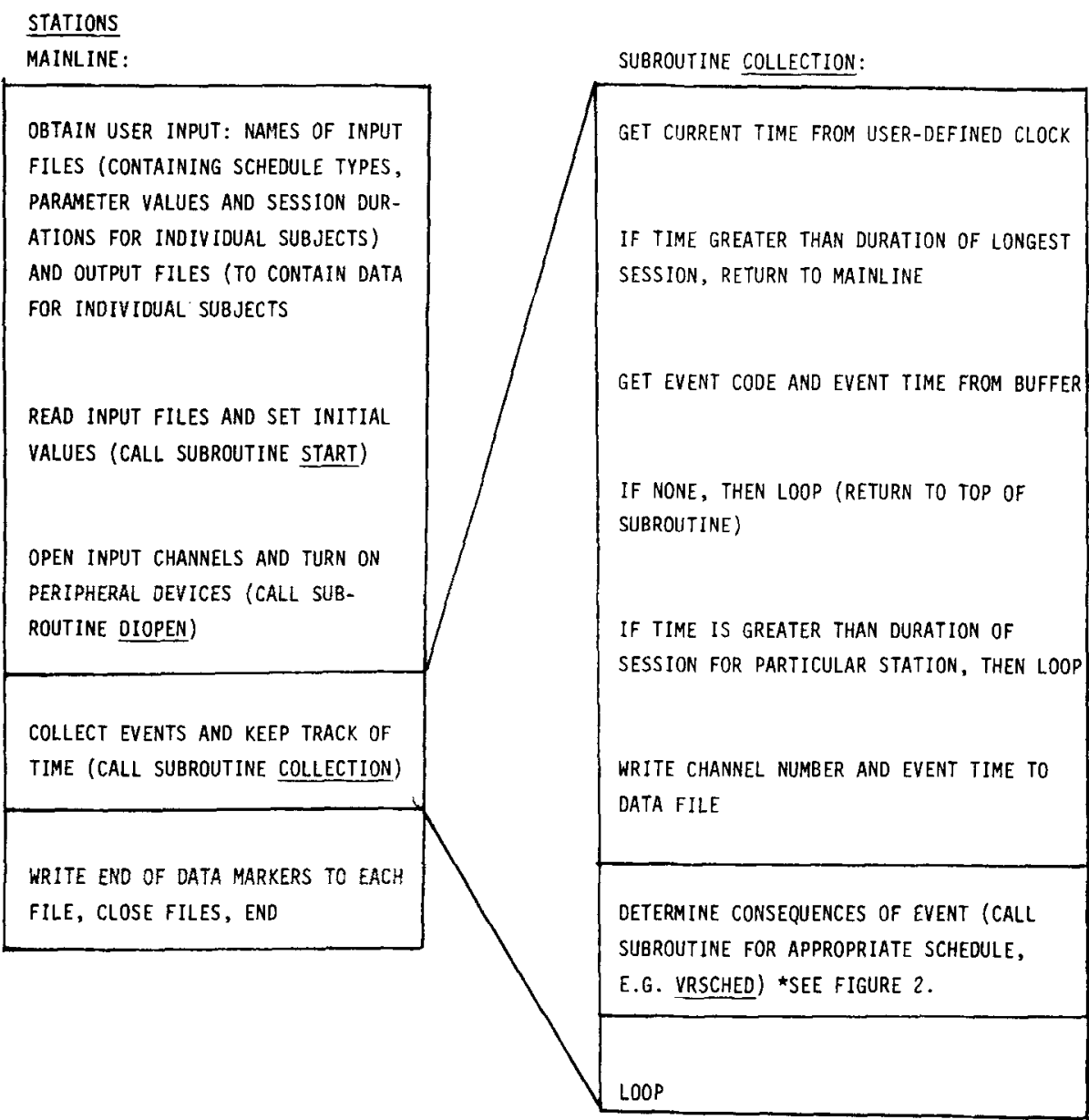

Figure 1. Schematic representation of the STATIONS mainline and the COLLECTION subroutine. 
The assembly language software is then interrupt driven, and it collects events and stores them in a buffer that is designed as a queue (first-in/first-out, or FIFO). FORTRAN programs may call the assembly language routine NEXTEVENT, which returns both the "oldest" ICODE (channel identifier) from the queue and the time at which the event occurred. The system is set up to be accurate within $.1 \mathrm{sec}$. However, it can be easily modified to millisecond accuracy, and with the addition of an external clock, 10-microsec accuracy can be achieved. A similar setup can be created easily and inexpensively for a variety of machines. We estimate $\$ 500$ for hardware and 2 days for assembly language programming.

Now we will describe the software structure, begin ning with the topmost, or mainline, level (see Figure 1). The mainline is concerned with the opening and closing of two types of files: (1) parameter files, which contain values for specific reinforcement schedules, [such as a list of interval values for a variable-interval (VI) schedule], that are then read into a VALUES array, and (2) data files (one per station) for storage of events and their times. In addition, the mainline passes control to the COLLECTION subroutine, which in turn collects events until the sessions are finished.

The sequence of events that are handled by the COLLECTION subroutine is also detailed in Figure 1. This routine is a software loop that scans for events until the sessions end. When an event is collected, control is passed to the appropriate type of reinforcement schedule routine (e.g., VRSCHED) based on specific event code (i.e., if a response occurs on the left key of Station 1, the subroutine that handles the type of reinforcement schedule being used for that key is invoked). A single variable-ratio (VR) subroutine, for example, handles all VR schedules for all keys in all boxes, regardless of differences between the specific schedule values in effect for the various keys.

The VRSCHED subroutine (see Figure 2) then checks an array that contains the current requirements for reinforcement for all schedules, the UNTILRFT array. To illustrate how this works, if the schedule associated with the left key of Station 1 is a VR

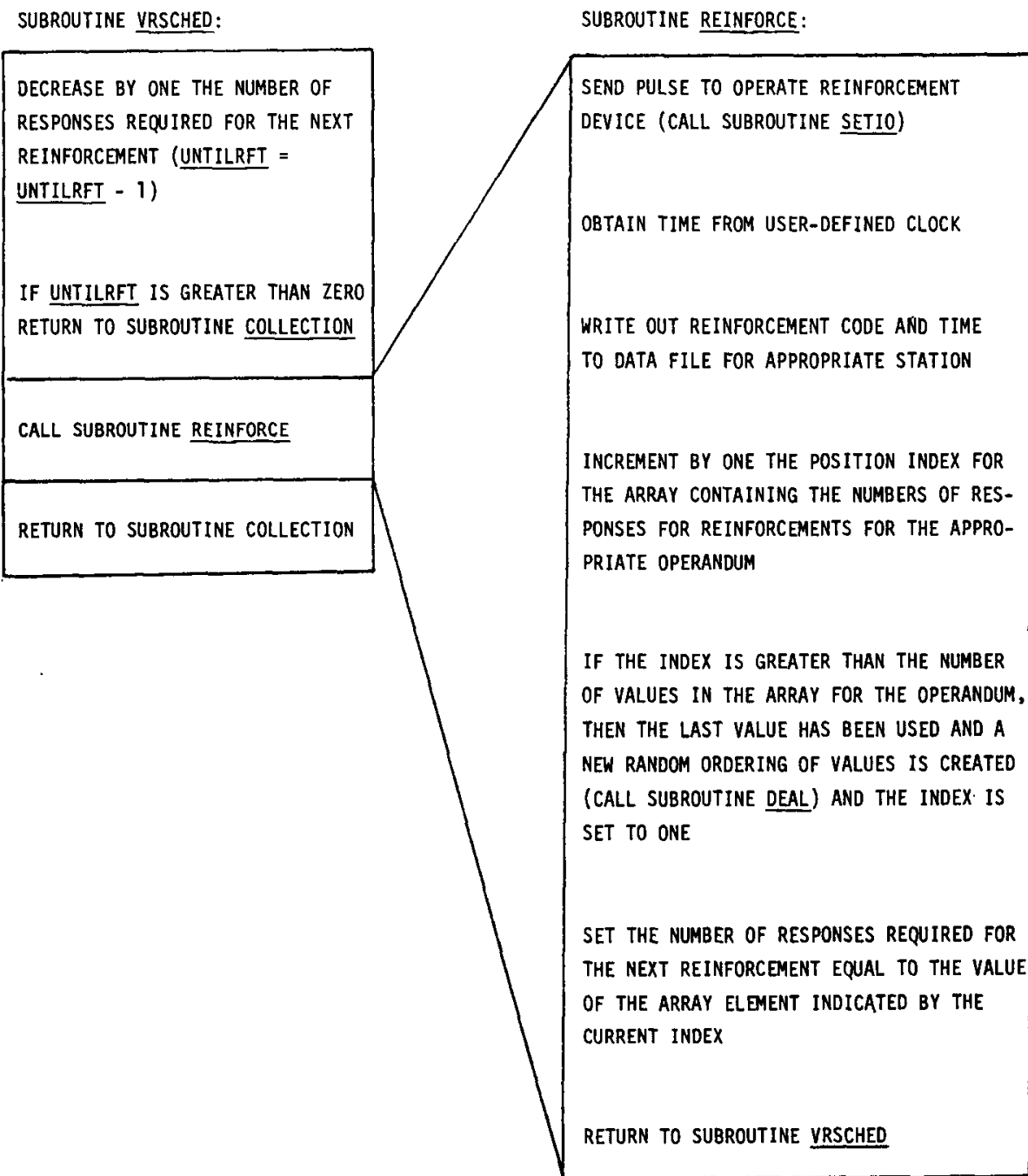

Figure 2. Schematic representation of the VRSCHED and REINFORCEMENT subroutines. 
schedule, the number of responses still required for reinforcement is held in the UNTILRFT array, and if the right-key schedule is a VI schedule, that position in the UNTILRFT array contains the time after which the next response would produce a reinforcement. Using the UNTILRFT array, then, the schedule subroutine determines whether or not the reinforcement requirement has been met. If not, control is passed back to the COLLECTION subroutine; otherwise, control is passed to the REINFORCE subroutine. Again, there is only one reinforcement routine that is called by all schedule routines.

The REINFORCE subroutine, also detailed in Figure 2, not only sends the signal to operate the reinforcement device but also records specific reinforcement delivery in the appropriate station's data file. Reinforcement duration is determined by peripheral logic. Control is passed back to the schedule routine, which then reads the next requirement for reinforcement for the appropriate schedule from the VALUES array in to the UNTILRFT array. Finally, control is returned to the COLLECTION routine.

The preceding description details the skeleton of the STATIONS software. Additional software features may be (and have been) easily appended. Because different schedule types are handled by separate routines, adding a new schedule type merely requires writing a single subroutine. In addition to several different schedule types, the current version of STATIONS also includes a changeover delay contingency (COD). The COD specifies the minimum amount of elapsed time since the beginning of a visit to a given key required for a response to be reinforced. Another contingency, such as a changeover ratio contingency (COR), could also be included with one additional subroutine. The COR would specify the minimum number of responses since the beginning of a visit to a key required for a response to be reinforced. The user could then select either a COD or COR, as well as the specific COD or COR value. Modifications of these types can often be performed in single FORTRAN routines and do not require any knowledge of assembly language. Thus the package is well suited to the psychologist with moderate programming skills who may be more interested in his research than in his equipment.

STATIONS is relatively simple and inexpensive to install and affords the user a substantial amount of control through easy modification. The top-down approach used in the design of the system results in both greater clarity of program structure and the possibility of local modifications without global repercussions. Thus top-down design and structured programming provide the cornerstone for the flexibility and portability that are STATIONS' strongest features.

\section{REFERENCES}

Brooks, F. P., JR. Mythical man-month: Essays on software engineering. New York: A \& W, 1974

Graнам, N. Introduction to computer science: A structured approach. St. Paul, Minn: West, 1979.

Kernigan, B. W., \& Plauger, P. J. Software tools. New York: $A \& W, 1976$. 